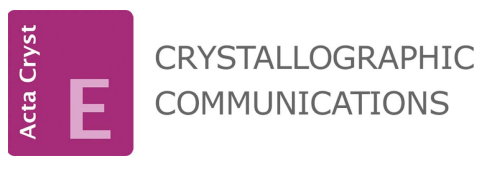

ISSN 2056-9890

Received 11 November 2021

Accepted 29 November 2021

Edited by A. V. Yatsenko, Moscow State University, Russia

Keywords: crystal structure; imidazo[1,2a]pyridine; hydrogen bonds; F...F contacts; Hirshfeld surface analysis.

CCDC reference: 2124974

Supporting information: this article has supporting information at journals.iucr.org/e

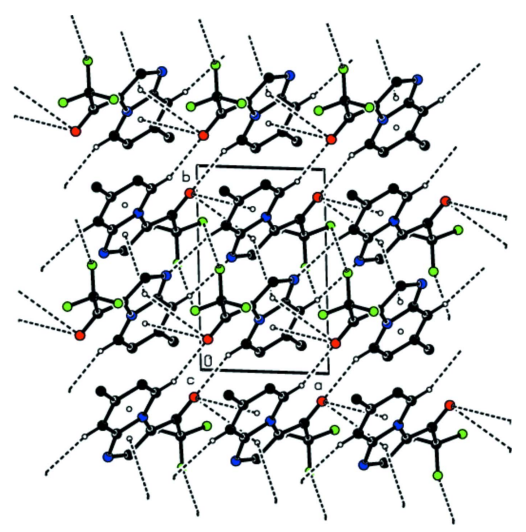

\section{Crystal structure and Hirshfeld surface analysis of 2,2,2-trifluoro-1-(7-methylimidazo[1,2-a]pyridin-3- yl)ethan-1-one}

\author{
Firudin I. Guseinov, ${ }^{\text {a,b }}$ Konstantin I. Kobrakov, ${ }^{a}$ Bogdan I. Ugrak, ${ }^{b}$ Zeliha Atioğlu, ${ }^{c}$ \\ Mehmet Akkurt ${ }^{\mathrm{d}}$ and Ajaya Bhattarai ${ }^{\mathrm{e}_{*}}$.
}

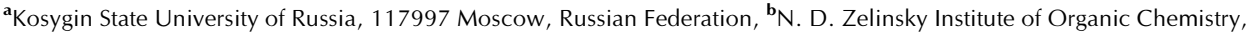
Russian Academy of Sciences, 119991 Moscow, Russian Federation, 'Department of Aircraft Electrics and Electronics, School of Applied Sciences, Cappadocia University, Mustafapaşa, 50420 Ürgüp, Nevşehir, Turkey, dDepartment of Physics, Faculty of Sciences, Erciyes University, 38039 Kayseri, Turkey, and ${ }^{\mathbf{e}}$ Department of Chemistry, M.M.A.M.C (Tribhuvan University) Biratnagar, Nepal. *Correspondence e-mail: bkajaya@yahoo.com
\end{abstract}

The bicyclic imidazo[1,2- $a$ ]pyridine core in the molecule of the title compound, $\mathrm{C}_{10} \mathrm{H}_{7} \mathrm{~F}_{3} \mathrm{~N}_{2} \mathrm{O}$, is planar within 0.004 (1) $\AA$. In the crystal, the molecules are linked by pairs of $\mathrm{C}-\mathrm{H} \cdots \mathrm{N}$ and $\mathrm{C}-\mathrm{H} \cdots \mathrm{O}$ hydrogen bonds, forming strips. These strips are connected by the F. .F contacts into layers, which are further joined by $\pi-\pi$ stacking interactions. The Hirshfeld surface analysis and fingerprint plots reveal that molecular packing is governed by $\mathrm{F} \cdots \mathrm{H} / \mathrm{H} \cdots \mathrm{F}$ $(31.6 \%), \mathrm{H} \cdots \mathrm{H}(16.8 \%), \mathrm{C} \cdots \mathrm{H} / \mathrm{H} \cdots \mathrm{C}(13.8 \%)$ and $\mathrm{O} \cdots \mathrm{H} / \mathrm{H} \cdots \mathrm{O}(8.5 \%)$ contacts.

\section{Chemical context}

The imidazo[1,2-a]pyridine synthon is one of the important fused bicyclic 5-6 heterocycles and it is recognized as a 'drug prejudice' scaffold because of its wide range of applications in medicinal chemistry (Bagdi et al., 2015). This synthon is also useful in coordination chemistry and catalysis because of its coordination ability and non-covalent donor-acceptor bonding (Guseinov et al., 2022; Ma et al., 2020, 2021; Mahmudov et al., 2020, 2021). Synthesis of this synthon from easily available chemicals is desirable due to its importance in the various branches of chemistry (Bagdi et al., 2015). Along with this, intermolecular interactions organize molecular architectures, which play a crucial role in synthesis, catalysis, micellization, etc. (Gurbanov et al., 2020a,b; Kopylovich et al., 2011; Ma et al., 2017a,b). The non-covalent bond-acceptor ability of both nitrogen atoms in the imidazo[1,2-a]pyridine synthon can be used in crystal engineering and in the design of dyes and other materials (Maharramov et al., 2018; Mizar et al., 2012; Shixaliyev et al., 2014; Shikhaliyev et al., 2018, 2019). Herein, we report a one-pot synthesis of 2,2,2-trifluoro-1-(7methylimidazo[1,2-a]pyridin-3-yl)ethan-1-one (I) from $(E / Z)$ 3-bromo-1,1,1-trifluoro-4-isopropoxybut-3-en-2-one and 4-methylpyridin-2-amine, which provides multiple intermolecular non-covalent interactions.<smiles>Cc1ccn2c(C(=O)C(F)(F)F)cnc2c1</smiles> 


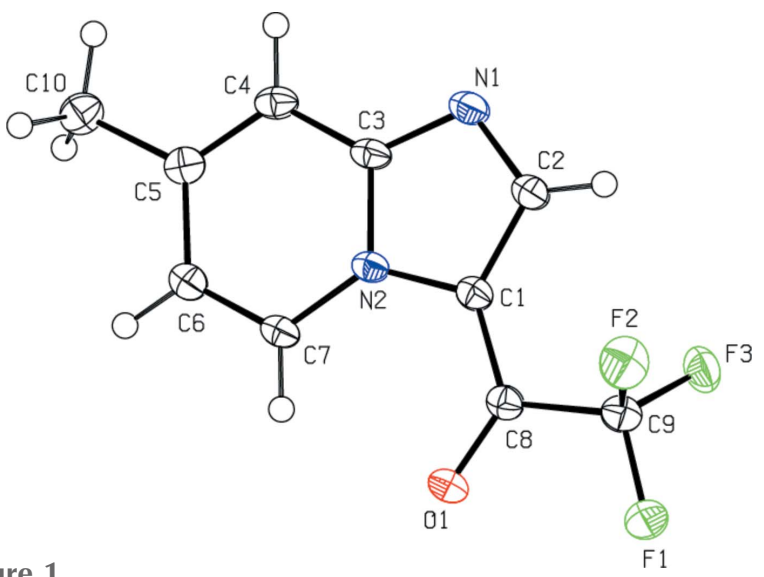

Figure 1

Molecular structure of the title compound showing the atom labelling and displacement ellipsoids drawn at the $50 \%$ probability level.

\section{Structural commentary}

In the molecule of the title compound (Fig. 1), the fused bicyclic imidazo[1,2-a]pyridine core is planar within 0.004 (1) $\AA$, with a dihedral angle of $0.34(6)^{\circ}$ between the mean planes of the five- and six-membered rings. The $\mathrm{C} 2-$ $\mathrm{C} 1-\mathrm{C} 8-\mathrm{C} 9$ and $\mathrm{N} 2-\mathrm{C} 1-\mathrm{C} 8-\mathrm{O} 1$ torsion angles of 1.04 (18) and $1.14(19)^{\circ}$, respectively, show that the ethanone group lies near the plane of the bicycle. The bond lengths $\mathrm{N} 1-\mathrm{C} 2, \mathrm{C} 2-\mathrm{C} 1$ and $\mathrm{C} 1-\mathrm{C} 8$ of 1.3367 (16), 1.3987 (16) and 1.4247 (16) $\AA$, respectively, indicate strong $\pi$-conjugation in the N1-O1 chain.

\section{Supramolecular features and Hirshfeld surface analysis}

In the crystal, the molecules are linked by pairs of $\mathrm{C}-\mathrm{H} \cdots \mathrm{N}$ and $\mathrm{C}-\mathrm{H} \cdots \mathrm{O}$ hydrogen bonds into strips elongated along the

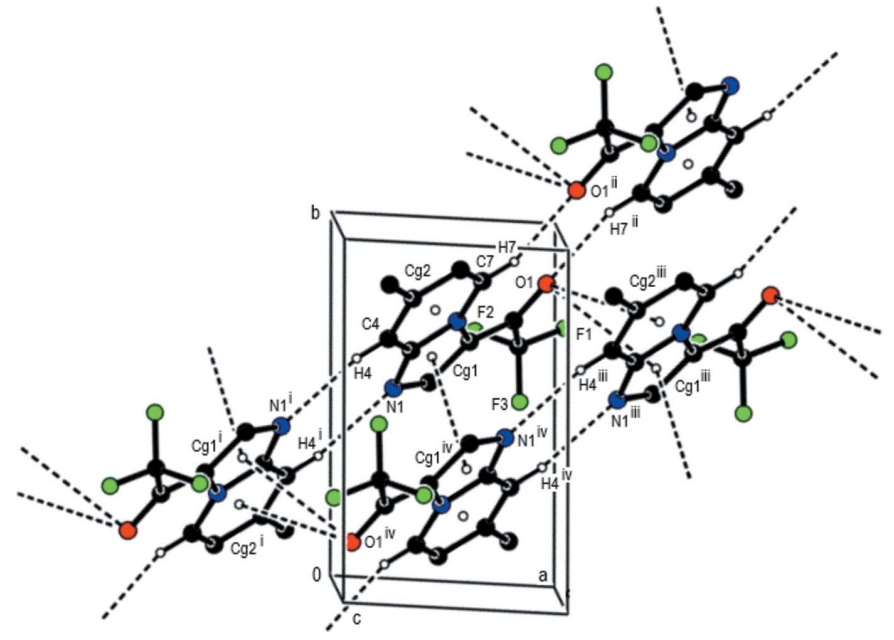

Figure 2

A general view of the $\mathrm{C}-\mathrm{H} \cdots \mathrm{O}$ and $\mathrm{C}-\mathrm{H} \cdots \mathrm{N}$ hydrogen bonds and $\pi-\pi$ stacking interactions in the title compound, depicted by dashed lines. Hydrogen atoms not involved in hydrogen bonding are omitted. [Symmetry codes: (i) $-x,-y+1,-z+1$; (ii) $-x+2,-y+2,-z+1$; (iii) $x+1, y, z$; (iv) $-1+x, y, z$.]
Table 1

Hydrogen-bond geometry $\left(\AA{ }^{\circ}{ }^{\circ}\right)$.

\begin{tabular}{lllll}
\hline$D-\mathrm{H} \cdots A$ & $D-\mathrm{H}$ & $\mathrm{H} \cdots A$ & $D \cdots A$ & $D-\mathrm{H} \cdots A$ \\
\hline $\mathrm{C} 4-\mathrm{H} 4 \cdots \mathrm{N} 1^{\mathrm{i}}$ & 0.95 & 2.48 & $3.4139(16)$ & 167 \\
$\mathrm{C} 7-\mathrm{H} 7 \cdots \mathrm{O} 1^{\mathrm{ii}}$ & 0.95 & 2.30 & $3.1464(14)$ & 147 \\
\hline
\end{tabular}

Symmetry codes: (i) $-x,-y+1,-z+1$; (ii) $-x+2,-y+2,-z+1$.

[210] direction (Figs. 2 and 3, Table 1). These strips are joined into layers parallel to $(1 \overline{2} 2)$ by $\mathrm{F}$...F contacts (Figs. 3-5, Table 2). The layers are connected by F...H contacts (Fig. 5, Table 2) and $\pi-\pi$ interactions with a shortest intercentroid separation of $3.6395(7) \AA[C g 1 \cdots C g 1(1-x, 1-y, 1-z)$; $C g 1$ is the centroid of the imidazole ring].

To visualize the intermolecular interactions in the title compound, the 3D Hirshfeld surfaces and two-dimensional fingerprint plots were computed using Crystal Explorer 17 (Turner et al., 2017). The Hirshfeld surface plotted over $d_{\text {norm }}$ in the range -0.3137 to 1.1314 a.u. is shown in Fig. 6. The intense red spots with negative $d_{\text {norm }}$ values represent $\mathrm{C}-$ $\mathrm{H} \cdots \mathrm{O}$ and $\mathrm{C}-\mathrm{H} \cdots \mathrm{N}$ hydrogen bonds. Pale red spots correspond to $\pi-\pi$ interactions, which are also seen in the shapeindex surface (Fig. 7) generated in the range -1 to $1 \AA$, where they are indicated by adjacent red and blue triangles. The

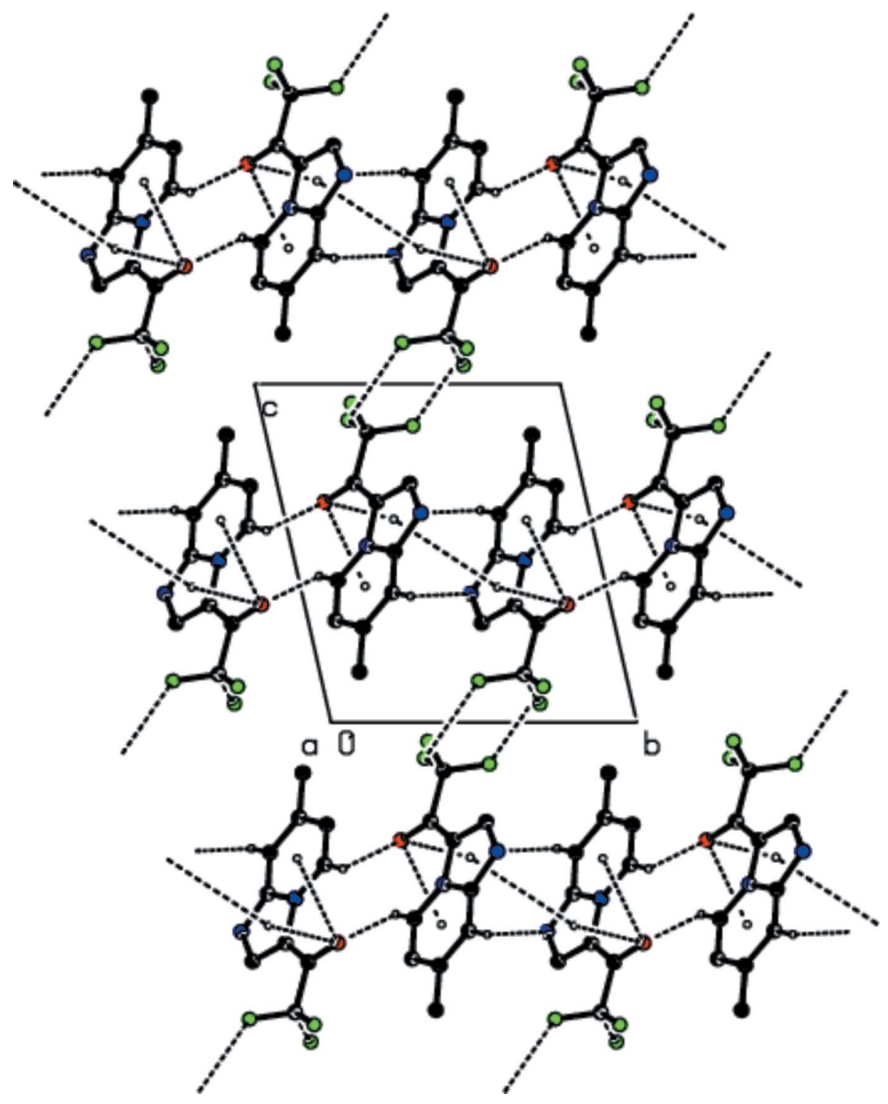

Figure 3

Packing diagram of the title compound, viewed down the $a$ axis showing the $\mathrm{C}-\mathrm{H} \cdots \mathrm{O}$ and $\mathrm{C}-\mathrm{H} \cdots \mathrm{N}$ hydrogen bonds and the $\mathrm{F} \cdots \mathrm{F}$ and $\pi-\pi$ stacking interactions. Hydrogen atoms not involved in hydrogen bonding are omitted. 


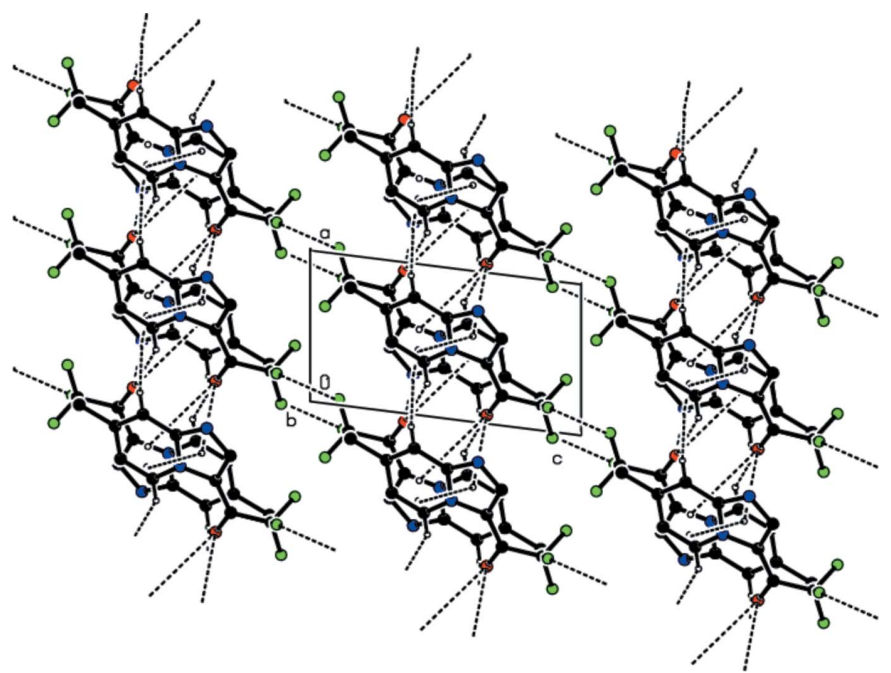

Figure 4

Packing diagram of the title compound, viewed down the $b$ axis showing the $\mathrm{C}-\mathrm{H} \cdots \mathrm{O}$ and $\mathrm{C}-\mathrm{H} \cdots \mathrm{N}$ hydrogen bonds and the $\mathrm{F} \cdots \mathrm{F}$ and $\pi-\pi$ stacking interactions. Hydrogen atoms not involved in hydrogen bonding are omitted.

Hirshfeld surface mapped over the electrostatic potential is shown in Fig. 8, where the hydrogen-bond acceptors are represented as red regions. The overall two-dimensional fingerprint plot, and those delineated into $\mathrm{F} \cdots \mathrm{H} / \mathrm{H} \cdots \mathrm{F}$ $(31.6 \%), \mathrm{H} \cdots \mathrm{H}(16.8 \%), \mathrm{C} \cdots \mathrm{H} / \mathrm{H} \cdots \mathrm{C}(13.8 \%)$ and $\mathrm{O} \cdots \mathrm{H} /$ $\mathrm{H} \cdots \mathrm{O}(8.5 \%)$ contacts are illustrated in Fig. 9. Other minor contributions to the Hirshfeld surface are from $\mathrm{N} \cdots \mathrm{H} / \mathrm{H} \cdots \mathrm{N}$ $(7.7 \%), \mathrm{F} \cdots \mathrm{F}(6.1 \%), \mathrm{O} \cdots \mathrm{C} / \mathrm{C} \cdots \mathrm{O}(4.2 \%), \mathrm{N} \cdots \mathrm{C} / \mathrm{C} \cdots \mathrm{N}$

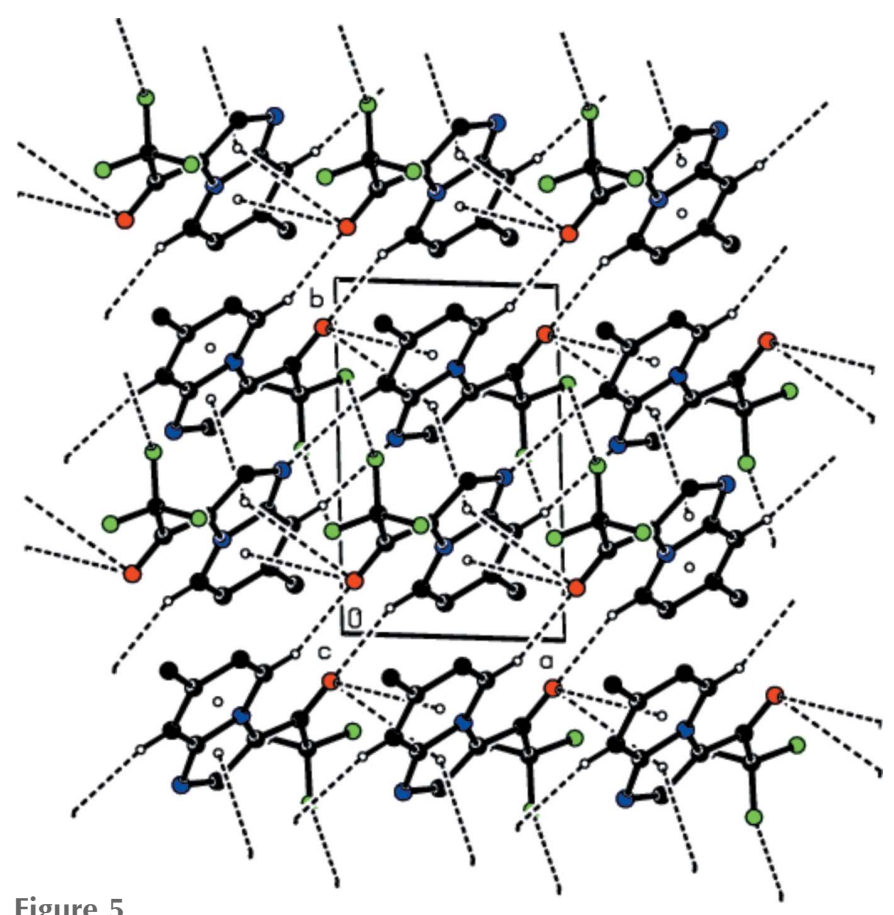

Figure 5

Packing diagram of the title compound, viewed down the $c$ axis showing the $\mathrm{C}-\mathrm{H} \cdots \mathrm{O}$ and $\mathrm{C}-\mathrm{H} \cdots \mathrm{N}$ hydrogen bonds and the $\mathrm{F} \cdots \mathrm{F}$ and $\pi-\pi$ stacking interactions. Hydrogen atoms not involved in hydrogen bonding are omitted.

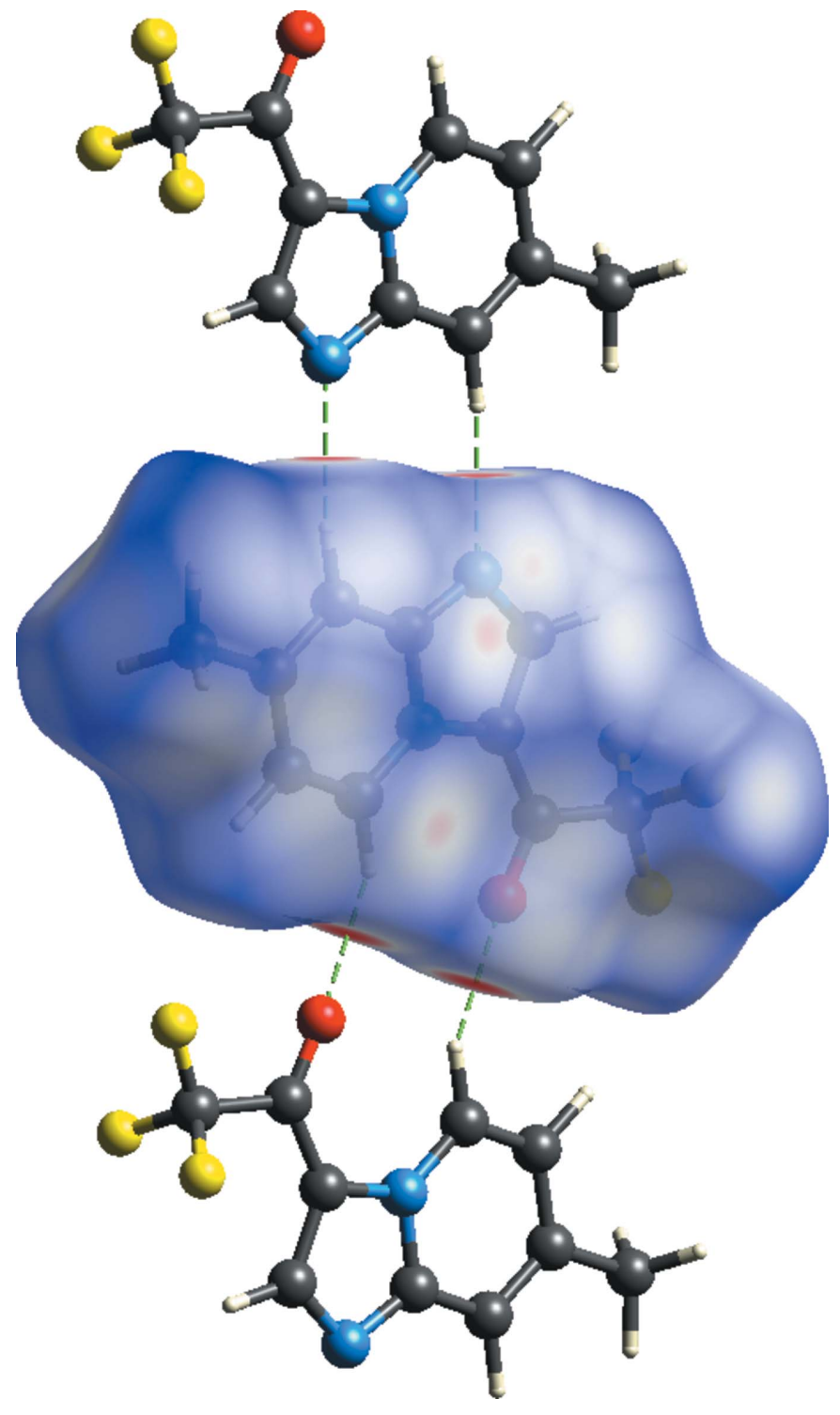

Figure 6

Hirshfeld surface of the title molecule mapped over $d_{\text {norm }}$.

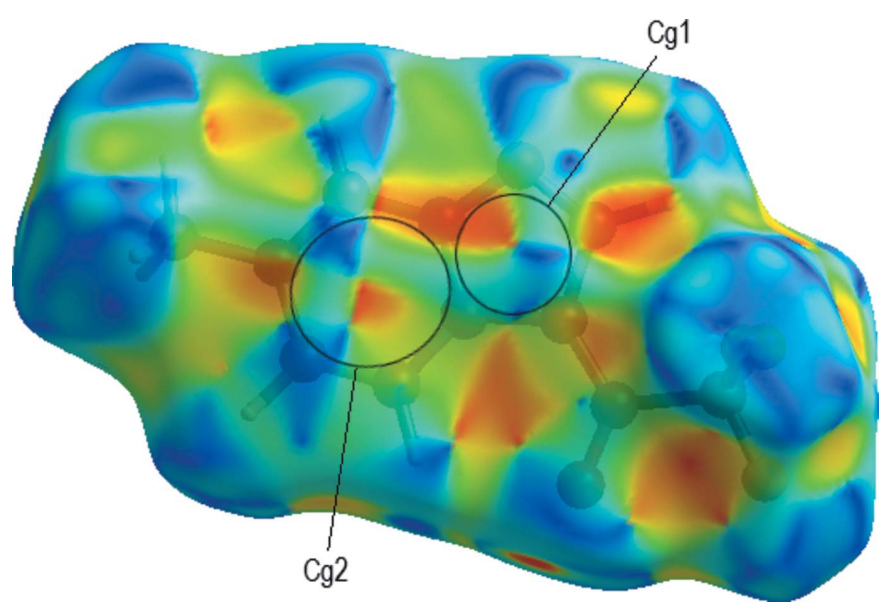

Figure 7

Hirshfeld surface of the title compound plotted over shape-index. 
Table 2

Summary of short interatomic contacts $(\AA)$ in the title structure.

\begin{tabular}{lll}
\hline Contact & Distance & Symmetry operation \\
\hline $\mathrm{O} 1 \cdots \mathrm{C} 3$ & $3.1574(15)$ & $1+x, y, z$ \\
$\mathrm{~F} 1 \cdots \mathrm{H} 10 B$ & 2.86 & $1+x, y,-1+z$ \\
$\mathrm{~F} 3 \cdots \mathrm{F} 1$ & $2.9074(11)$ & $2-x, 1-y,-z$ \\
$\mathrm{H} 10 \mathrm{C} \cdots \mathrm{O} 1$ & 2.83 & $1-x, 2-y, 1-z$ \\
$\mathrm{~F} 2 \cdots \mathrm{H} 10 A$ & 2.64 & $x, y,-1+z$ \\
$\mathrm{H} 2 \cdots \mathrm{F} 2$ & 2.80 & $1-x, 1-y,-z$ \\
$\mathrm{C} 3 \cdots \mathrm{N} 1$ & $3.3055(16)$ & $1-x, 1-y, 1-z$ \\
\hline
\end{tabular}

$(3.8 \%), \quad \mathrm{C} \cdots \mathrm{C}(2.4 \%), \quad \mathrm{F} \cdots \mathrm{C} / \mathrm{C} \cdots \mathrm{F} \quad(1.7 \%), \quad \mathrm{F} \cdots \mathrm{N} / \mathrm{N} \cdots \mathrm{F}$ $(1.4 \%), \mathrm{N} \cdots \mathrm{N}(1.1 \%)$ and $\mathrm{O} \cdots \mathrm{N} / \mathrm{N} \cdots \mathrm{O}(0.9 \%)$ contacts.

\section{Database survey}

The most closely related compounds containing a similar imidazo[1,2-a]pyridine skeleton, but with different substituents on the amide $\mathrm{N}$ atom are: $N$-t-butyl-2-(phenylethynyl)imidazo[1,2-a]pyridin-3-amine (XOWVOX; Tber et al., 2019), 6-bromo-2-(4-bromophenyl)imidazo[1,2-a]pyridine (KOXGEM; Khamees et al., 2019), $N$-t-butyl-2-(2-nitrophenyl)imidazo[1,2-a]pyridin-3-amine (PILGAV01; Dhanalakshmi et al., 2019), 2-(4-methoxyphenyl)-6-nitroimidazo[1,2-a]pyridine-3-carbaldehyde (DABTEI; Koudad et al., 2015), 2-(ethylsulfinyl)imidazo[1,2-a]pyridine-3-sulfonamide (ZAPJAD; Gong et al., 2012) and 2-methyl-6-(trifluoromethyl)imidazo[1,2-a]pyridine-3-carbonitrile (ULEGOI; Fun et al., 2011). In the crystal of XOWVOX, molecules are linked by $\mathrm{N}-\mathrm{H} \cdots \mathrm{H}$ hydrogen bonds, forming chains along the $c$-axis direction. The chains are linked by $\mathrm{C}-\mathrm{H} \cdots \pi$ interactions, forming slabs parallel to the $a c$ plane. In the structure of KOXGEM, an intramolecular $\mathrm{C}-\mathrm{H} \cdots \mathrm{N}$ hydrogen bond forms an $S(5)$ ring motif. In the crystal, a short $\mathrm{H} \cdot \cdots \mathrm{H}$ contact

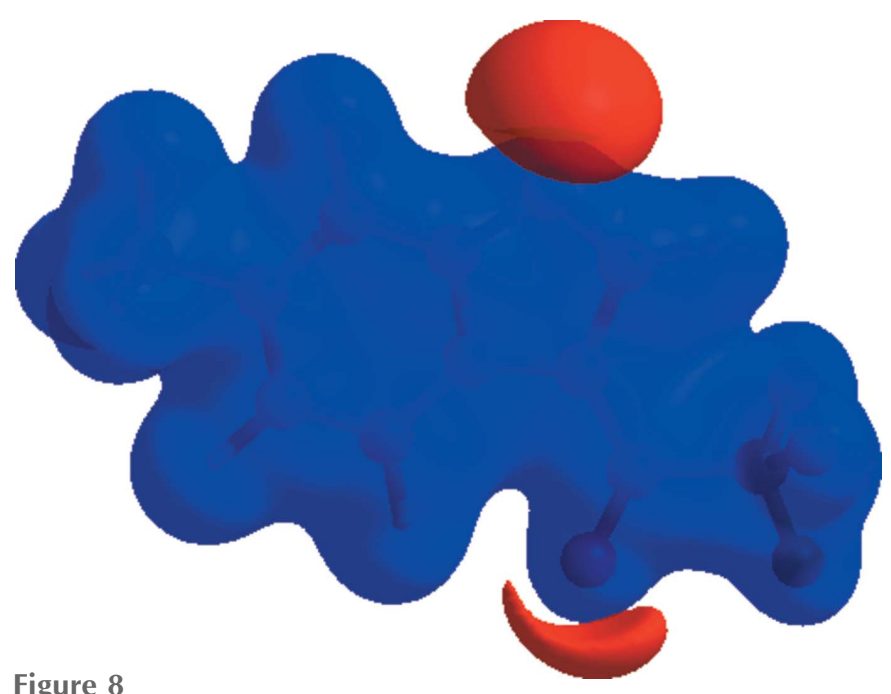

Figure 8

View of the three-dimensional Hirshfeld surface of the title molecule plotted over electrostatic potential energy in the range -0.0500 to 0.0500 a.u. calculated at the Hartree-Fock level of theory using the STO-3 $G$ basis set. Hydrogen-bond donors and acceptors are shown as blue and red regions around the atoms, corresponding to positive and negative potentials, respectively.

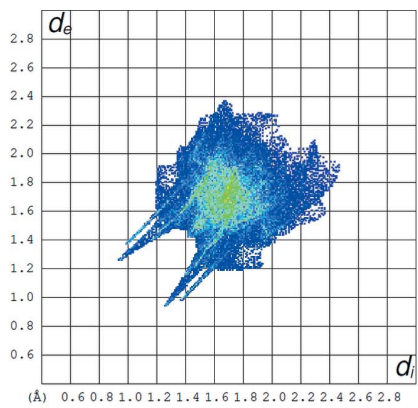

(a) All...All

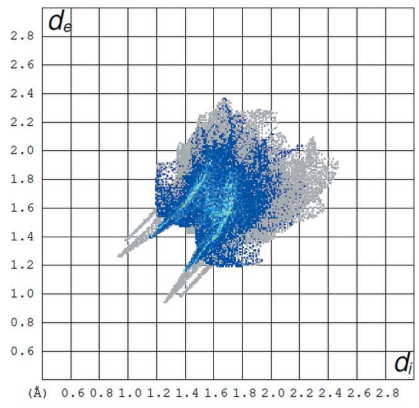

(b) $\mathbf{F} \cdots \mathbf{H} / \mathbf{H} \cdots \mathbf{F}$

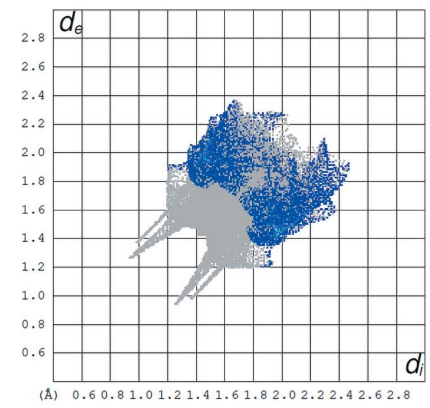

(d) $\mathbf{C} \cdots \mathbf{H} / \mathbf{H} \cdots \mathbf{C}$

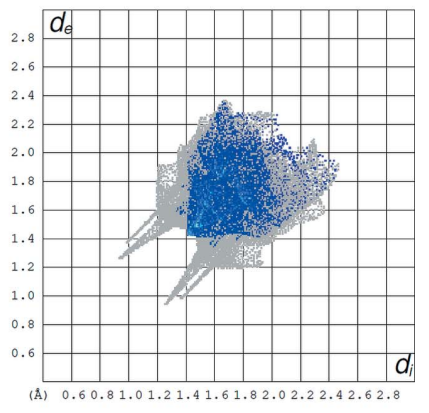

(c) $\mathrm{H} . . . \mathrm{H}$

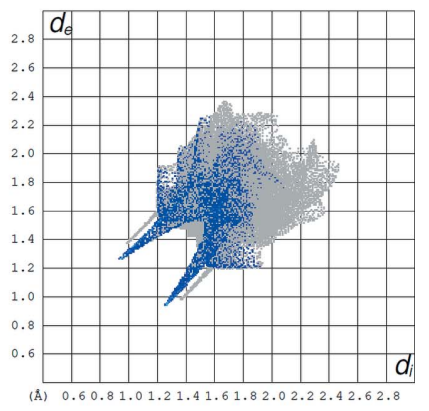

(e) $\mathbf{O} \cdots \mathbf{H} / \mathbf{H} \cdots \mathbf{O}$
Figure 9

(a) The overall two-dimensional fingerprint plot and those delineated into $(b) \mathrm{F} \cdots \mathrm{H} / \mathrm{H} \cdots \mathrm{F},(c) \mathrm{H} \cdots \mathrm{H},(d) \mathrm{C} \cdots \mathrm{H} / \mathrm{H} \cdots \mathrm{C}$ and $(e) \mathrm{O} \cdots \mathrm{H} / \mathrm{H} \cdots \mathrm{O}$ interactions.

links adjacent molecules into centrosymmetric dimers. The dimers are joined by weak $\mathrm{C}-\mathrm{H} \cdots \pi$ and slipped $\pi-\pi$ stacking interactions, forming layers parallel to (110), which are connected into a three-dimensional network by short $\mathrm{Br} \cdots \mathrm{H}$ contacts. In the crystal of PILGAV01, N-H $\cdots \mathrm{N}$ hydrogen bonds link the molecules into [010] chains. The cohesion of the crystal structure of DABTEI is ensured by $\mathrm{C}-\mathrm{H} \cdots \mathrm{N}$ and $\mathrm{C}-$ $\mathrm{H}$. . O hydrogen bonds, forming layers parallel to the $a c$ plane. In ZAPJAD, the supramolecular structure is defined by two kinds of intermolecular hydrogen bonds. Pairs of $\mathrm{N}-\mathrm{H} \cdots \mathrm{N}$ hydrogen bonds link the molecules into centrosymmetric dimers and $\mathrm{N}-\mathrm{H} \cdots \mathrm{O}$ hydrogen bonds link the dimers into tubular chains running along the $a$-axis direction. In the crystal of ULEGOI, molecules are linked into chains through pairs of $\mathrm{C}-\mathrm{H} \cdots \mathrm{N}$ interactions, forming $R_{2}^{2}(12)$ and $R_{2}^{2}(8)$ hydrogenbond ring motifs. These chains are stacked along the $a$ axis. 
Table 3

Experimental details.

\begin{tabular}{|c|c|}
\hline \multicolumn{2}{|l|}{ Crystal data } \\
\hline Chemical formula & $\mathrm{C}_{10} \mathrm{H}_{7} \mathrm{~F}_{3} \mathrm{~N}_{2} \mathrm{O}$ \\
\hline$M_{\mathrm{r}}$ & 228.18 \\
\hline Crystal system, space group & Triclinic, $P \overline{1}$ \\
\hline Temperature $(\mathrm{K})$ & 100 \\
\hline$a, b, c(\AA)$ & $5.4384(1), 8.8298(2), 10.0744(2)$ \\
\hline$\alpha, \beta, \gamma\left({ }^{\circ}\right)$ & 102.501 (2), 96.764 (2), 91.415 (2) \\
\hline$V\left(\AA^{3}\right)$ & $468.39(2)$ \\
\hline$Z$ & 2 \\
\hline Radiation type & $\mathrm{Cu} K \alpha$ \\
\hline$\mu\left(\mathrm{mm}^{-1}\right)$ & 1.30 \\
\hline Crystal size $(\mathrm{mm})$ & $0.15 \times 0.06 \times 0.02$ \\
\hline \multicolumn{2}{|l|}{ Data collection } \\
\hline Diffractometer & XtaLAB Synergy, Dualflex, HyPix \\
\hline Absorption correction & $\begin{array}{l}\text { Multi-scan (CrysAlis PRO; Rigaku } \\
\text { OD, 2021) }\end{array}$ \\
\hline$T_{\min }, T_{\max }$ & $0.323,1.000$ \\
\hline $\begin{array}{l}\text { No. of measured, independent and } \\
\text { observed }[I>2 \sigma(I)] \text { reflections }\end{array}$ & $14165,2006,1927$ \\
\hline$R_{\text {int }}$ & 0.050 \\
\hline$(\sin \theta / \lambda)_{\max }\left(\AA^{-1}\right)$ & 0.638 \\
\hline \multicolumn{2}{|l|}{ Refinement } \\
\hline$R\left[F^{2}>2 \sigma\left(F^{2}\right)\right], w R\left(F^{2}\right), S$ & $0.037,0.105,1.09$ \\
\hline No. of reflections & 2006 \\
\hline No. of parameters & 147 \\
\hline H-atom treatment & H-atom parameters constrained \\
\hline$\Delta \rho_{\max }, \Delta \rho_{\min }\left(\mathrm{e} \AA^{-3}\right)$ & $0.37,-0.26$ \\
\hline
\end{tabular}

Computer programs: CrysAlis PRO (Rigaku OD, 2021), SHELXT (Sheldrick, 2015a), SHELXL (Sheldrick, 2015b), ORTEP-3 for Windows (Farrugia, 2012) and PLATON (Spek, 2020).

\section{Synthesis and crystallization}

A mixture of $(E / Z)$-3-bromo-1,1,1-trifluoro-4-isopropoxybut3-en-2-one (0.522 mg, $2 \mathrm{mmol})$ and 4-methylpyridin-2-amine $(0.216 \mathrm{mg}, 2 \mathrm{mmol})$ in dry isopropyl alcohol $(15 \mathrm{~mL})$ was refluxed for $4 \mathrm{~h}$. Then the solvent was removed on a rotary evaporator under reduced pressure. The residue was recrystallized from methanol. Crystals suitable for X-ray analysis were obtained by slow evaporation of a methanol solution. Colourless solid (yield 94\%), m.p. 405-406 K. Analysis calculated for $\mathrm{C}_{10} \mathrm{H}_{7} \mathrm{~F}_{3} \mathrm{~N}_{2} \mathrm{O}(M=228.17)$ : C 52.64, $\mathrm{H} 3.09, \mathrm{~N}$ 12.28; found: C 52.55, H 3.07, N 12.19\%. ${ }^{1} \mathrm{H}$ NMR $(300 \mathrm{MHz}$, $\left.\mathrm{CDCl}_{3}\right) \delta 2.48\left(3 \mathrm{H}, \mathrm{CH}_{3}\right), 7.32-8.60(3 \mathrm{H}, \mathrm{Ar}), 9.32(1 \mathrm{H}, \mathrm{CH})$. ${ }^{13} \mathrm{C} \mathrm{NMR}\left(75 \mathrm{MHz}, \mathrm{CDCl}_{3}\right) \delta 174.45,150.22,141.32,135.67$, 131.04, 123.55, 118.66, 118.21, 116.32 and 21.56. ESI-MS: $\mathrm{m} / z$ : $229.18[M+\mathrm{H}]^{+}$.

\section{Refinement}

Crystal data, data collection and structure refinement details are summarized in Table 3. Hydrogen atoms were positioned geometrically $(\mathrm{C}-\mathrm{H}=0.95-0.98 \AA)$ and refined as riding, with $U_{\text {iso }}(\mathrm{H})=1.2 U_{\text {eq }}(\mathrm{C})$ for $\mathrm{CH}$ hydrogen atoms and $U_{\text {iso }}(\mathrm{H})=$ $1.5 U_{\text {eq }}(\mathrm{C})$ for $\mathrm{CH}_{3}$ hydrogen atoms.

\section{Acknowledgements}

The author's contributions are as follows. Conceptualization, FIG, MA and AB; synthesis, FIG and KIK; X-ray analysis,
BIU, ZA and MA; writing (review and editing of the manuscript), FIG, ZA, MA and AB.

\section{References}

Bagdi, A. K., Santra, S., Monir, K. \& Hajra, A. (2015). Chem. Commun. 51, 1555-1575.

Dhanalakshmi, G., Mala, R., Thennarasu, S. \& Aravindhan, S. (2019). IUCrData, 4, x191477.

Farrugia, L. J. (2012). J. Appl. Cryst. 45, 849-854.

Fun, H.-K., Rosli, M. M., Kumar, D. J. M., Prasad, D. J. \& Nagaraja, G. K. (2011). Acta Cryst. E67, o573.

Gong, Y., Ma, H. \& Li, J. (2012). Acta Cryst. E68, o1342.

Gurbanov, A. V., Kuznetsov, M. L., Demukhamedova, S. D., Alieva, I. N., Godjaev, N. M., Zubkov, F. I., Mahmudov, K. T. \& Pombeiro, A. J. L. (2020a). CrystEngComm, 22, 628-633.

Gurbanov, A. V., Kuznetsov, M. L., Mahmudov, K. T., Pombeiro, A. J. L. \& Resnati, G. (2020b). Chem. Eur. J. 26, 14833-14837.

Guseinov, F. I., Malinnikov, V. M., Lialin, K. N., Kobrakov, K. I., Shuvalova, E. V., Nelyubina, Y. V., Ugrak, B. I., Kustov, L. M. \& Mahmudov, K. T. (2022). Dyes Pigments, 197, 109898.

Khamees, H. A., Chaluvaiah, K., El-khatatneh, N. A., Swamynayaka, A., Chong, K. H., Dasappa, J. P. \& Madegowda, M. (2019). Acta Cryst. E75, 1620-1626.

Kopylovich, M. N., Mahmudov, K. T., Mizar, A. \& Pombeiro, A. J. L. (2011). Chem. Commun. 47, 7248-7250.

Koudad, M., Elaatiaoui, A., Benchat, N., Saadi, M. \& El Ammari, L. (2015). Acta Cryst. E71, o979-o980.

Ma, Z., Gurbanov, A. V., Maharramov, A. M., Guseinov, F. I., Kopylovich, M. N., Zubkov, F. I., Mahmudov, K. T. \& Pombeiro, A. J. L. (2017a). J. Mol. Catal. A Chem. 426, 526-533.

Ma, Z., Gurbanov, A. V., Sutradhar, M., Kopylovich, M. N., Mahmudov, K. T., Maharramov, A. M., Guseinov, F. I., Zubkov, F. I. \& Pombeiro, A. J. L. (2017b). Mol. Catal. 428, 17-23.

Ma, Z., Mahmudov, K. T., Aliyeva, V. A., Gurbanov, A. V., Guedes da Silva, M. F. C. \& Pombeiro, A. J. L. (2021). Coord. Chem. Rev. 437, 213859.

Ma, Z., Mahmudov, K. T., Aliyeva, V. A., Gurbanov, A. V. \& Pombeiro, A. J. L. (2020). Coord. Chem. Rev. 423, 213482.

Maharramov, A. M., Shikhaliyev, N. Q., Suleymanova, G. T., Gurbanov, A. V., Babayeva, G. V., Mammadova, G. Z., Zubkov, F. I., Nenajdenko, V. G., Mahmudov, K. T. \& Pombeiro, A. J. L. (2018). Dyes Pigments, 159, 135-141.

Mahmudov, K. T., Gurbanov, A. V., Aliyeva, V. A., Resnati, G. \& Pombeiro, A. J. L. (2020). Coord. Chem. Rev. 418, 213381.

Mahmudov, K. T., Huseynov, F. E., Aliyeva, V. A., Guedes da Silva, M. F. C. \& Pombeiro, A. J. L. (2021). Chem. Eur. J. 27, 1437014389.

Mizar, A., Guedes da Silva, M. F. C., Kopylovich, M. N., Mukherjee, S., Mahmudov, K. T. \& Pombeiro, A. J. L. (2012). Eur. J. Inorg. Chem. 2012, 2305-2313.

Rigaku OD (2021). CrysAlis PRO. Rigaku Oxford Diffraction, Tokyo, Japan.

Sheldrick, G. M. (2015a). Acta Cryst. A71, 3-8.

Sheldrick, G. M. (2015b). Acta Cryst. C71, 3-8.

Shikhaliyev, N. Q., Ahmadova, N. E., Gurbanov, A. V., Maharramov, A. M., Mammadova, G. Z., Nenajdenko, V. G., Zubkov, F. I., Mahmudov, K. T. \& Pombeiro, A. J. L. (2018). Dyes Pigments, 150, 377-381.

Shikhaliyev, N. Q., Kuznetsov, M. L., Maharramov, A. M., Gurbanov, A. V., Ahmadova, N. E., Nenajdenko, V. G., Mahmudov, K. T. \& Pombeiro, A. J. L. (2019). CrystEngComm, 21, 5032-5038.

Shixaliyev, N. Q., Gurbanov, A. V., Maharramov, A. M., Mahmudov, K. T., Kopylovich, M. N., Martins, L. M. D. R. S., Muzalevskiy, V. M., Nenajdenko, V. G. \& Pombeiro, A. J. L. (2014). New J. Chem. 38, 4807-4815. 
Spek, A. L. (2020). Acta Cryst. E76, 1-11.

Tber, Z., Kansiz, S., El Hafi, M., Loubidi, M., Jouha, J., Dege, N., Essassi, E. M. \& Mague, J. T. (2019). Acta Cryst. E75, 15641567.
Turner, M. J., Mckinnon, J. J., Wolff, S. K., Grimwood, D. J., Spackman, P. R., Jayatilaka, D. \& Spackman, M. A. (2017). Crystal Explorer 17. The University of Western Australia. http://hirshfeldsurface.net 


\section{supporting information}

Acta Cryst. (2022). E78, 12-17 [https://doi.org/10.1107/S2056989021012676]

\section{Crystal structure and Hirshfeld surface analysis of 2,2,2-trifluoro-1-(7-methyl- imidazo[1,2-a]pyridin-3-yl)ethan-1-one}

Firudin I. Guseinov, Konstantin I. Kobrakov, Bogdan I. Ugrak, Zeliha Atioğlu, Mehmet Akkurt and Ajaya Bhattarai

Computing details

Data collection: CrysAlis PRO (Rigaku OD, 2021); cell refinement: CrysAlis PRO (Rigaku OD, 2021); data reduction: CrysAlis PRO (Rigaku OD, 2021); program(s) used to solve structure: SHELXT (Sheldrick, 2015a); program(s) used to refine structure: SHELXL (Sheldrick, 2015b); molecular graphics: ORTEP-3 for Windows (Farrugia, 2012); software used to prepare material for publication: PLATON (Spek, 2020).

2,2,2-Trifluoro-1-(7-methylimidazo[1,2-a]pyridin-3-yl)ethan-1-one

Crystal data

$\mathrm{C}_{10} \mathrm{H}_{7} \mathrm{~F}_{3} \mathrm{~N}_{2} \mathrm{O}$

$M_{r}=228.18$

Triclinic, $P \overline{1}$

$a=5.4384$ (1) $\AA$

$b=8.8298(2) \AA$

$c=10.0744(2) \AA$

$\alpha=102.501(2)^{\circ}$

$\beta=96.764(2)^{\circ}$

$\gamma=91.415(2)^{\circ}$

$V=468.39(2) \AA^{3}$

\section{Data collection}

XtaLAB Synergy, Dualflex, HyPix diffractometer

Radiation source: micro-focus sealed X-ray tube, PhotonJet $(\mathrm{Cu}) \mathrm{X}$-ray Source

Mirror monochromator

Detector resolution: 10.0000 pixels $\mathrm{mm}^{-1}$

$\omega$ scans

Absorption correction: multi-scan

(CrysAlis PRO; Rigaku OD, 2021)

Refinement

Refinement on $F^{2}$

Least-squares matrix: full

$R\left[F^{2}>2 \sigma\left(F^{2}\right)\right]=0.037$

$w R\left(F^{2}\right)=0.105$

$S=1.09$

2006 reflections
$Z=2$

$F(000)=232$

$D_{\mathrm{x}}=1.618 \mathrm{Mg} \mathrm{m}^{-3}$

$\mathrm{Cu} K \alpha$ radiation, $\lambda=1.54184 \AA$

Cell parameters from 11472 reflections

$\theta=4.5-79.1^{\circ}$

$\mu=1.30 \mathrm{~mm}^{-1}$

$T=100 \mathrm{~K}$

Block, colorless

$0.15 \times 0.06 \times 0.02 \mathrm{~mm}$

$T_{\min }=0.323, T_{\max }=1.000$

14165 measured reflections

2006 independent reflections

1927 reflections with $I>2 \sigma(I)$

$R_{\text {int }}=0.050$

$\theta_{\max }=79.5^{\circ}, \theta_{\min }=4.5^{\circ}$

$h=-6 \rightarrow 6$

$k=-11 \rightarrow 10$

$l=-12 \rightarrow 12$

147 parameters

0 restraints

Primary atom site location: dual

Hydrogen site location: inferred from neighbouring sites

$\mathrm{H}$-atom parameters constrained 
$w=1 /\left[\sigma^{2}\left(F_{\mathrm{o}}^{2}\right)+(0.0617 P)^{2}+0.1479 P\right]$

where $P=\left(F_{\mathrm{o}}^{2}+2 F_{\mathrm{c}}{ }^{2}\right) / 3$

$(\Delta / \sigma)_{\max }=0.001$

$\Delta \rho_{\max }=0.37 \mathrm{e} \AA^{-3}$

$\Delta \rho_{\min }=-0.26$ e $\AA^{-3}$

Special details

Geometry. All esds (except the esd in the dihedral angle between two 1.s. planes) are estimated using the full covariance matrix. The cell esds are taken into account individually in the estimation of esds in distances, angles and torsion angles; correlations between esds in cell parameters are only used when they are defined by crystal symmetry. An approximate (isotropic) treatment of cell esds is used for estimating esds involving l.s. planes.

Fractional atomic coordinates and isotropic or equivalent isotropic displacement parameters $\left(\AA^{2}\right)$

\begin{tabular}{lllll}
\hline & $x$ & $y$ & $z$ & $U_{\text {iso }} * / U_{\mathrm{eq}}$ \\
\hline F3 & $0.83669(15)$ & $0.51338(8)$ & $0.12281(8)$ & $0.0298(2)$ \\
F1 & $1.03655(15)$ & $0.72062(9)$ & $0.10632(8)$ & $0.0306(2)$ \\
F2 & $0.63947(16)$ & $0.69737(10)$ & $0.05569(8)$ & $0.0352(2)$ \\
O1 & $0.94235(16)$ & $0.85798(10)$ & $0.34833(9)$ & $0.0231(2)$ \\
N2 & $0.53525(17)$ & $0.75163(11)$ & $0.47862(10)$ & $0.0178(2)$ \\
N1 & $0.25848(19)$ & $0.55184(12)$ & $0.38082(11)$ & $0.0224(2)$ \\
C3 & $0.3285(2)$ & $0.66590(13)$ & $0.49404(12)$ & $0.0195(3)$ \\
C7 & $0.6410(2)$ & $0.87311(13)$ & $0.57981(12)$ & $0.0199(3)$ \\
H7 & 0.783693 & 0.930322 & 0.567582 & $0.024^{*}$ \\
C6 & $0.5364(2)$ & $0.90977(14)$ & $0.69840(12)$ & $0.0214(3)$ \\
H6 & 0.608051 & 0.993640 & 0.769395 & $0.026^{*}$ \\
C4 & $0.2206(2)$ & $0.70392(14)$ & $0.61568(13)$ & $0.0214(3)$ \\
H4 & 0.077951 & 0.646052 & 0.626999 & $0.026^{*}$ \\
C5 & $0.3224(2)$ & $0.82541(14)$ & $0.71850(13)$ & $0.0211(3)$ \\
C2 & $0.4210(2)$ & $0.56623(14)$ & $0.29402(12)$ & $0.0210(3)$ \\
H2 & 0.416403 & 0.500889 & 0.205342 & $0.025^{*}$ \\
C1 & $0.5986(2)$ & $0.68799(13)$ & $0.34799(12)$ & $0.0191(3)$ \\
C9 & $0.8297(2)$ & $0.66755(14)$ & $0.14284(13)$ & $0.0231(3)$ \\
C8 & $0.7991(2)$ & $0.74800(13)$ & $0.29223(12)$ & $0.0190(3)$ \\
C10 & $0.2118(2)$ & $0.87010(16)$ & $0.85047(13)$ & $0.0267(3)$ \\
H10A & 0.336148 & 0.863035 & 0.927312 & $0.040^{*}$ \\
H10B & 0.068569 & 0.799545 & 0.848113 & $0.040^{*}$ \\
H10C & 0.158430 & 0.976875 & 0.862058 & $0.040^{*}$ \\
& & & & \\
\hline
\end{tabular}

(Sheldrick 2015b),

$\mathrm{Fc}^{*}=\mathrm{kFc}\left[1+0.001 \mathrm{xFc}^{2} \lambda^{3} / \sin (2 \theta)\right]^{-1 / 4}$

Extinction coefficient: 0.0061 (14)
Extinction correction: SHELXL2018/3

Atomic displacement parameters $\left(\AA^{2}\right)$

\begin{tabular}{lllllll}
\hline & $U^{11}$ & $U^{22}$ & $U^{33}$ & $U^{12}$ & $U^{13}$ & $U^{23}$ \\
\hline F3 & $0.0391(5)$ & $0.0189(4)$ & $0.0298(4)$ & $-0.0012(3)$ & $0.0086(3)$ & $-0.0001(3)$ \\
F1 & $0.0318(4)$ & $0.0315(4)$ & $0.0291(4)$ & $-0.0052(3)$ & $0.0120(3)$ & $0.0047(3)$ \\
F2 & $0.0349(5)$ & $0.0452(5)$ & $0.0241(4)$ & $0.0054(4)$ & $-0.0037(3)$ & $0.0081(3)$ \\
O1 & $0.0223(4)$ & $0.0190(4)$ & $0.0270(4)$ & $-0.0048(3)$ & $0.0026(3)$ & $0.0037(3)$ \\
N2 & $0.0161(5)$ & $0.0153(5)$ & $0.0222(5)$ & $-0.0015(3)$ & $0.0010(4)$ & $0.0058(4)$ \\
N1 & $0.0197(5)$ & $0.0184(5)$ & $0.0280(5)$ & $-0.0036(4)$ & $0.0008(4)$ & $0.0044(4)$ \\
C3 & $0.0158(5)$ & $0.0162(5)$ & $0.0272(6)$ & $-0.0019(4)$ & $0.0002(4)$ & $0.0079(4)$
\end{tabular}


supporting information

\begin{tabular}{lllllll} 
C7 & $0.0180(5)$ & $0.0167(5)$ & $0.0241(6)$ & $-0.0029(4)$ & $-0.0006(4)$ & $0.0051(4)$ \\
C6 & $0.0208(6)$ & $0.0192(6)$ & $0.0231(6)$ & $-0.0002(4)$ & $-0.0002(4)$ & $0.0044(4)$ \\
C4 & $0.0177(5)$ & $0.0206(6)$ & $0.0282(6)$ & $-0.0006(4)$ & $0.0026(4)$ & $0.0110(5)$ \\
C5 & $0.0201(6)$ & $0.0203(6)$ & $0.0250(6)$ & $0.0029(4)$ & $0.0023(4)$ & $0.0098(5)$ \\
C2 & $0.0196(6)$ & $0.0180(6)$ & $0.0240(6)$ & $-0.0017(4)$ & $0.0000(4)$ & $0.0034(4)$ \\
C1 & $0.0191(6)$ & $0.0167(5)$ & $0.0213(6)$ & $-0.0006(4)$ & $0.0008(4)$ & $0.0046(4)$ \\
C9 & $0.0228(6)$ & $0.0225(6)$ & $0.0244(6)$ & $-0.0014(4)$ & $0.0025(5)$ & $0.0065(5)$ \\
C8 & $0.0186(5)$ & $0.0162(5)$ & $0.0227(6)$ & $0.0010(4)$ & $0.0009(4)$ & $0.0063(4)$ \\
C10 & $0.0278(6)$ & $0.0282(7)$ & $0.0264(6)$ & $0.0017(5)$ & $0.0054(5)$ & $0.0097(5)$ \\
\hline
\end{tabular}

Geometric parameters $\left(A,{ }^{\circ}\right)$

\begin{tabular}{|c|c|c|c|}
\hline $\mathrm{F} 3-\mathrm{C} 9$ & $1.3345(14)$ & $\mathrm{C} 6-\mathrm{H} 6$ & 0.9500 \\
\hline $\mathrm{F} 1-\mathrm{C} 9$ & $1.3292(14)$ & $\mathrm{C} 6-\mathrm{C} 5$ & $1.4245(17)$ \\
\hline $\mathrm{F} 2-\mathrm{C} 9$ & $1.3453(14)$ & $\mathrm{C} 4-\mathrm{H} 4$ & 0.9500 \\
\hline $\mathrm{O} 1-\mathrm{C} 8$ & $1.2208(15)$ & $\mathrm{C} 4-\mathrm{C} 5$ & $1.3728(18)$ \\
\hline $\mathrm{N} 2-\mathrm{C} 3$ & $1.3827(14)$ & $\mathrm{C} 5-\mathrm{C} 10$ & $1.5028(17)$ \\
\hline $\mathrm{N} 2-\mathrm{C} 7$ & $1.3706(15)$ & $\mathrm{C} 2-\mathrm{H} 2$ & 0.9500 \\
\hline $\mathrm{N} 2-\mathrm{C} 1$ & $1.4011(15)$ & $\mathrm{C} 2-\mathrm{C} 1$ & $1.3987(16)$ \\
\hline $\mathrm{N} 1-\mathrm{C} 3$ & $1.3571(16)$ & $\mathrm{C} 1-\mathrm{C} 8$ & $1.4247(16)$ \\
\hline $\mathrm{N} 1-\mathrm{C} 2$ & $1.3367(16)$ & $\mathrm{C} 9-\mathrm{C} 8$ & $1.5489(17)$ \\
\hline $\mathrm{C} 3-\mathrm{C} 4$ & $1.3997(17)$ & $\mathrm{C} 10-\mathrm{H} 10 \mathrm{~A}$ & 0.9800 \\
\hline $\mathrm{C} 7-\mathrm{H} 7$ & 0.9500 & $\mathrm{C} 10-\mathrm{H} 10 \mathrm{~B}$ & 0.9800 \\
\hline $\mathrm{C} 7-\mathrm{C} 6$ & $1.3629(17)$ & $\mathrm{C} 10-\mathrm{H} 10 \mathrm{C}$ & 0.9800 \\
\hline $\mathrm{C} 3-\mathrm{N} 2-\mathrm{C} 1$ & $106.60(10)$ & $\mathrm{N} 1-\mathrm{C} 2-\mathrm{C} 1$ & $112.65(11)$ \\
\hline $\mathrm{C} 7-\mathrm{N} 2-\mathrm{C} 3$ & $122.01(10)$ & $\mathrm{C} 1-\mathrm{C} 2-\mathrm{H} 2$ & 123.7 \\
\hline $\mathrm{C} 7-\mathrm{N} 2-\mathrm{C} 1$ & $131.39(10)$ & $\mathrm{N} 2-\mathrm{C} 1-\mathrm{C} 8$ & $123.53(11)$ \\
\hline $\mathrm{C} 2-\mathrm{N} 1-\mathrm{C} 3$ & $105.23(10)$ & $\mathrm{C} 2-\mathrm{C} 1-\mathrm{N} 2$ & $104.25(10)$ \\
\hline $\mathrm{N} 2-\mathrm{C} 3-\mathrm{C} 4$ & $119.63(11)$ & $\mathrm{C} 2-\mathrm{C} 1-\mathrm{C} 8$ & $132.19(11)$ \\
\hline $\mathrm{N} 1-\mathrm{C} 3-\mathrm{N} 2$ & $111.28(10)$ & $\mathrm{F} 3-\mathrm{C} 9-\mathrm{F} 2$ & $106.95(10)$ \\
\hline $\mathrm{N} 1-\mathrm{C} 3-\mathrm{C} 4$ & $129.10(11)$ & $\mathrm{F} 3-\mathrm{C} 9-\mathrm{C} 8$ & $113.36(9)$ \\
\hline $\mathrm{N} 2-\mathrm{C} 7-\mathrm{H} 7$ & 120.8 & $\mathrm{~F} 1-\mathrm{C} 9-\mathrm{F} 3$ & $107.82(10)$ \\
\hline $\mathrm{C} 6-\mathrm{C} 7-\mathrm{N} 2$ & $118.35(11)$ & $\mathrm{F} 1-\mathrm{C} 9-\mathrm{F} 2$ & $107.29(10)$ \\
\hline $\mathrm{C} 6-\mathrm{C} 7-\mathrm{H} 7$ & 120.8 & $\mathrm{~F} 1-\mathrm{C} 9-\mathrm{C} 8$ & $110.72(10)$ \\
\hline $\mathrm{C} 7-\mathrm{C} 6-\mathrm{H} 6$ & 119.2 & $\mathrm{~F} 2-\mathrm{C} 9-\mathrm{C} 8$ & $110.45(10)$ \\
\hline $\mathrm{C} 7-\mathrm{C} 6-\mathrm{C} 5$ & $121.61(11)$ & $\mathrm{O} 1-\mathrm{C} 8-\mathrm{C} 1$ & $126.81(11)$ \\
\hline $\mathrm{C} 5-\mathrm{C} 6-\mathrm{H} 6$ & 119.2 & $\mathrm{O} 1-\mathrm{C} 8-\mathrm{C} 9$ & $117.43(10)$ \\
\hline $\mathrm{C} 3-\mathrm{C} 4-\mathrm{H} 4$ & 120.2 & $\mathrm{C} 1-\mathrm{C} 8-\mathrm{C} 9$ & $115.72(10)$ \\
\hline $\mathrm{C} 5-\mathrm{C} 4-\mathrm{C} 3$ & $119.55(11)$ & $\mathrm{C} 5-\mathrm{C} 10-\mathrm{H} 10 \mathrm{~A}$ & 109.5 \\
\hline $\mathrm{C} 5-\mathrm{C} 4-\mathrm{H} 4$ & 120.2 & $\mathrm{C} 5-\mathrm{C} 10-\mathrm{H} 10 \mathrm{~B}$ & 109.5 \\
\hline $\mathrm{C} 6-\mathrm{C} 5-\mathrm{C} 10$ & $119.98(11)$ & $\mathrm{C} 5-\mathrm{C} 10-\mathrm{H} 10 \mathrm{C}$ & 109.5 \\
\hline $\mathrm{C} 4-\mathrm{C} 5-\mathrm{C} 6$ & $118.86(11)$ & $\mathrm{H} 10 \mathrm{~A}-\mathrm{C} 10-\mathrm{H} 10 \mathrm{~B}$ & 109.5 \\
\hline $\mathrm{C} 4-\mathrm{C} 5-\mathrm{C} 10$ & $121.17(11)$ & $\mathrm{H} 10 \mathrm{~A}-\mathrm{C} 10-\mathrm{H} 10 \mathrm{C}$ & 109.5 \\
\hline $\mathrm{N} 1-\mathrm{C} 2-\mathrm{H} 2$ & 123.7 & $\mathrm{H} 10 \mathrm{~B}-\mathrm{C} 10-\mathrm{H} 10 \mathrm{C}$ & 109.5 \\
\hline $\mathrm{F} 3-\mathrm{C} 9-\mathrm{C} 8-\mathrm{O} 1$ & $130.29(11)$ & $\mathrm{C} 3-\mathrm{N} 1-\mathrm{C} 2-\mathrm{C} 1$ & $0.16(13)$ \\
\hline $\mathrm{F} 3-\mathrm{C} 9-\mathrm{C} 8-\mathrm{C} 1$ & $-51.85(14)$ & $\mathrm{C} 3-\mathrm{C} 4-\mathrm{C} 5-\mathrm{C} 6$ & $-0.07(16)$ \\
\hline
\end{tabular}




\begin{tabular}{llll}
$\mathrm{F} 1-\mathrm{C} 9-\mathrm{C} 8-\mathrm{O} 1$ & $8.98(15)$ & $\mathrm{C} 3-\mathrm{C} 4-\mathrm{C} 5-\mathrm{C} 10$ & $179.86(10)$ \\
$\mathrm{F} 1-\mathrm{C} 9-\mathrm{C} 8-\mathrm{C} 1$ & $-173.16(10)$ & $\mathrm{C} 7-\mathrm{N} 2-\mathrm{C} 3-\mathrm{N} 1$ & $-179.78(10)$ \\
$\mathrm{F} 2-\mathrm{C} 9-\mathrm{C} 8-\mathrm{O} 1$ & $-109.71(12)$ & $\mathrm{C} 7-\mathrm{N} 2-\mathrm{C} 3-\mathrm{C} 4$ & $0.59(16)$ \\
$\mathrm{F} 2-\mathrm{C} 9-\mathrm{C} 8-\mathrm{C} 1$ & $68.15(13)$ & $\mathrm{C} 7-\mathrm{N} 2-\mathrm{C} 1-\mathrm{C} 2$ & $179.87(11)$ \\
$\mathrm{N} 2-\mathrm{C} 3-\mathrm{C} 4-\mathrm{C} 5$ & $-0.35(17)$ & $\mathrm{C} 7-\mathrm{N} 2-\mathrm{C} 1-\mathrm{C} 8$ & $-2.02(19)$ \\
$\mathrm{N} 2-\mathrm{C} 7-\mathrm{C} 6-\mathrm{C} 5$ & $-0.07(17)$ & $\mathrm{C} 7-\mathrm{C} 6-\mathrm{C} 5-\mathrm{C} 4$ & $0.29(17)$ \\
$\mathrm{N} 2-\mathrm{C} 1-\mathrm{C} 8-\mathrm{O} 1$ & $1.14(19)$ & $\mathrm{C} 7-\mathrm{C} 6-\mathrm{C} 5-\mathrm{C} 10$ & $-179.65(10)$ \\
$\mathrm{N} 2-\mathrm{C} 1-\mathrm{C} 8-\mathrm{C} 9$ & $-176.48(9)$ & $\mathrm{C} 2-\mathrm{N} 1-\mathrm{C} 3-\mathrm{N} 2$ & $-0.18(13)$ \\
$\mathrm{N} 1-\mathrm{C} 3-\mathrm{C} 4-\mathrm{C} 5$ & $-179.91(11)$ & $\mathrm{C} 2-\mathrm{N} 1-\mathrm{C} 3-\mathrm{C} 4$ & $179.41(11)$ \\
$\mathrm{N} 1-\mathrm{C} 2-\mathrm{C} 1-\mathrm{N} 2$ & $-0.09(13)$ & $\mathrm{C} 2-\mathrm{C} 1-\mathrm{C} 8-\mathrm{O} 1$ & $178.67(12)$ \\
$\mathrm{N} 1-\mathrm{C} 2-\mathrm{C} 1-\mathrm{C} 8$ & $-177.96(12)$ & $\mathrm{C} 2-\mathrm{C} 1-\mathrm{C} 8-\mathrm{C} 9$ & $1.04(18)$ \\
$\mathrm{C} 3-\mathrm{N} 2-\mathrm{C} 7-\mathrm{C} 6$ & $-0.37(16)$ & $\mathrm{C} 1-\mathrm{N} 2-\mathrm{C} 3-\mathrm{N} 1$ & $0.13(13)$ \\
$\mathrm{C} 3-\mathrm{N} 2-\mathrm{C} 1-\mathrm{C} 2$ & $-0.03(12)$ & $\mathrm{C} 1-\mathrm{N} 2-\mathrm{C} 3-\mathrm{C} 4$ & $-179.50(9)$ \\
$\mathrm{C} 3-\mathrm{N} 2-\mathrm{C} 1-\mathrm{C} 8$ & $178.08(10)$ & $\mathrm{C} 1-\mathrm{N} 2-\mathrm{C} 7-\mathrm{C} 6$ & $179.75(11)$ \\
\hline
\end{tabular}

Hydrogen-bond geometry $\left(A,{ }^{\circ}\right)$

\begin{tabular}{lllll}
\hline$D-\mathrm{H} \cdots A$ & $D-\mathrm{H}$ & $\mathrm{H} \cdots A$ & $D \cdots A$ & $D-\mathrm{H} \cdots A$ \\
\hline $\mathrm{C} 2-\mathrm{H} 2 \cdots \mathrm{F} 3$ & 0.95 & 2.53 & $2.9876(14)$ & 110 \\
$\mathrm{C} 4-\mathrm{H} 4 \cdots \mathrm{N} 1^{\mathrm{i}}$ & 0.95 & 2.48 & $3.4139(16)$ & 167 \\
$\mathrm{C} 7-\mathrm{H} 7 \cdots \mathrm{O} 1$ & 0.95 & 2.43 & $2.9864(14)$ & 117 \\
$\mathrm{C} 7-\mathrm{H} 7 \cdots \mathrm{O} 1^{\mathrm{ii}}$ & 0.95 & 2.30 & $3.1464(14)$ & 147 \\
\hline
\end{tabular}

Symmetry codes: (i) $-x,-y+1,-z+1$; (ii) $-x+2,-y+2,-z+1$. 